\title{
Glutathione Oxidation Is Associated With Airway Macrophage Functional Impairment in Children With Severe Asthma
}

\author{
ANNE M. FITZPATRICK, W. GERALD TEAGUE, LEANDREA BURWELL, MEREDITH S. BROWN, AND LOU ANN S. BROWN; \\ FOR THE NIH/NHLBI SEVERE ASTHMA RESEARCH PROGRAM
}

Department of Pediatrics, Emory University School of Medicine, Atlanta, Georgia 30322

\begin{abstract}
Airway cellular dysfunction is a differentiating feature of severe asthma in children that may be related to an imbalance of the antioxidant, glutathione (GSH). We hypothesized that oxidation of GSH to glutathione disulfide (GSSG) in the epithelial lining fluid (ELF) of children with severe asthma would contribute to altered airway macrophage (AM) GSH homeostasis and AM cellular dysfunction. Bronchoalveolar lavage (BAL) was performed in 64 asthmatic children (severe asthma, $n=43$ ). GSH, GSSG, markers of lipid peroxidation and DNA oxidation, and IL-8 were quantified in the BAL supernatant. GSH, GSSG, activities of histone deacetylase (HDAC) and histone acetyltransferase, apoptosis, and phagocytosis were assessed in isolated AMs. Children with severe asthma had increased GSSG, lipid peroxidation, byproducts of DNA oxidation, and inflammation in the ELF. This imbalance of GSH homeostasis was also noted intracellularly within the AMs and was associated with decreased HDAC activities, increased apoptosis, and impaired phagocytosis. In vitro GSH supplementation inhibited apoptosis and rescued phagocytosis in children with severe asthma. Severe asthma in children is characterized by altered airway and intracellular AM GSH homeostasis that translates to impaired AM function. Interventions to restore airway GSH homeostasis may be warranted in children with severe asthma. (Pediatr Res 69: 154-159, 2011)
\end{abstract}

$\mathrm{S}^{\mathrm{e}}$ evere or "difficult-to-treat" asthma in children is a complicated disorder associated with a high degree of morbidity. Although the pathophysiology of severe asthma in children is not entirely understood, symptoms are thought to result from persistent airway inflammation and oxidant stress that are not attenuated with corticosteroid treatment $(1,2)$. This unopposed cycle of airway inflammation and oxidant stress ultimately contributes to irreversible airway injury (3), which further increases the frequency and severity of symptoms (1).

Glutathione (GSH) is a thiol antioxidant that is highly expressed in the epithelial lining fluid (ELF) and within airway cells $(4,5)$, where it functions as a regulator of the cell cycle and enzyme substrate (6). With exposure to hydroperoxides, GSH becomes oxidized, forming glutathione disulfide (GSSG). Although acute changes in the GSH to GSSG ratio are necessary for the detoxification of reactive oxygen species, chronic GSSG formation can inhibit the antioxidant capabilities of the airways through depletion of the total GSH pool

Received May 27, 2010; accepted September 9, 2010.

Correspondence: Anne M. Fitzpatrick, Ph.D., 2015 Uppergate Drive, Atlanta, GA 30322; e-mail: anne.fitzpatrick@emory.edu

Supported by Grants KO1 NR010548, RO1 HL69170, the Children's Healthcare of Atlanta Center for Developmental Lung Biology, and Grant UL1 RR025008 from the Clinical and Translational Science Award Program
(7). This altered ratio of GSH to GSSG further promotes histone acetylation by increasing histone acetyltransferase (HAT) activity and inhibiting histone deacetylase (HDAC) activity in airway cells, leading to enhanced release of proinflammatory cytokines, such as IL-8 (8). This mechanism may account for the airway hyperresponsiveness and dysfunction previously observed in ovalbumin-sensitized mice, which is abrogated with GSH supplementation and restoration of the GSH/GSSG balance in airway tissue (9).

Although the precise role of GSH in asthma severity is unclear, low GSH and increased GSSG concentrations have been reported in the exhaled breath (10) and ELF (2) of asthmatic children. Although corticosteroid treatment normalizes the airway GSH to GSSG ratio in mild-to-moderate asthmatic children with acute exacerbations (10), children with severe asthma have marked depletion of GSH, increased GSSG formation, and increased lipid peroxidation in the airways despite high-dose corticosteroid therapy (2). Given the vital role of GSH in maintaining cellular processes, altered airway GSH homeostasis in children with severe asthma may have profound implications for vital airway functions, including innate immune defenses against foreign pathogens (11). Indeed, we have previously observed significant alterations of airway macrophage (AM) function in children with severe asthma, including increased proinflammatory cytokine and chemokine release, increased apoptosis, and impaired phagocytosis of infectious particles (12). Therefore, the purpose of this study was to quantify GSH homeostasis in both the ELF and AMs of children with severe versus moderate asthma. We hypothesized that increased oxidation of GSH in the ELF would result in a shift in the AM intracellular GSH to GSSG ratio and AM cellular dysfunction.

\section{METHODS}

Sample. This study was approved by the Emory University Institutional Review Board. Informed consent was obtained from all participating children and their legal guardians. Children also provided written and verbal assent. Recruitment was limited to children, 6-18 y of age with physician-diagnosed asthma undergoing bronchoscopy for clinical indications. All children had persistent asthma (13) with a history of at least a $12 \%$ change in the forced 
expiratory volume in 1 second $\left(\mathrm{FEV}_{1}\right)$ after bronchodilator administration. All children were treated with a stable dose of inhaled corticosteroid (ICS) for at least $6 \mathrm{wk}$ before enrollment. Severe asthma was diagnosed according to criteria developed by the National Heart, Lung and Blood Institute's Severe Asthma Research Program (1), which were adapted from the American Thoracic Society's Workshop Report (14). Severe asthma was diagnosed in children treated with high-dose ICS or daily oral corticosteroids who also had two minor criteria (1). Thresholds for high-dose ICS were defined as $\geq 440 \mu \mathrm{g}$ of fluticasone equivalent per day for children $<12$ y and $\geq 880 \mu \mathrm{g}$ for children, 12-18 y of age. Children who did not meet criteria for severe asthma were classified as having moderate asthma given the extent of symptoms and current treatment with ICS (13).

For diagnostic purposes, children with moderate and severe asthma underwent flexible bronchoscopy with bronchoalveolar lavage (BAL) as indicated for persistent asthma symptoms despite ICS treatment. Seven children with moderate asthma also underwent bronchoscopy for suspected foreign body aspiration $(n=2)$ and recurrent pneumonia $(n=5)$. All children were clinically stable at the time of bronchoscopy with no evidence of an acute upper respiratory tract infection, including no rhinorrhea and no increase in chest symptoms or short-acting bronchodilator use for 2 wk before the procedure. The BAL return volume was divided between the research and clinical laboratories. The samples submitted to the clinical laboratories were subjected to standard culture and sensitivity testing, viral respiratory panels, and cytopathological stains for bacteria and fungi.

Procedures. Spirometry was performed with a portable spirometer (KoKo Legend, Ferraris, Louisville, CO) (15), and the best of three forced vital capacity (FVC) maneuvers was interpreted (16). Venipuncture was performed immediately before bronchoscopy. Bronchoscopy was performed under general anesthesia using a laryngeal mask airway and flexible bronchoscope (Olympus BF-3C160; Olympus America Inc., Melville, NY). BAL fluid was collected from the right middle lobe with three $1 \mathrm{~mL} / \mathrm{kg}$ (50 mL maximum) saline lavages.

Blood and BAL samples were centrifuged at 5000 and $1200 \mathrm{rpm}$, respectively, for 7-10 min. To prevent auto-oxidation before GSH and GSSG analysis, $250 \mu \mathrm{L}$ of BAL supernatant was immediately added to an equal volume of $5 \%$ perchloric acid preservation solution containing iodoacetic acid $(6.7 \mu \mathrm{M})$ and boric acid $(0.1 \mathrm{~mol} / \mathrm{L})$ with $5 \mu \mathrm{M} \gamma$-glutamyl-glutamate and immediately frozen at $-80^{\circ} \mathrm{C}(17)$.

Total cell counts were performed with a hemocytometer, and cellular differentials were determined from 300 consecutive cells after Diff-Quick staining. Cell pellets with $<85 \%$ AM purity were excluded from further analysis. AMs were purified from the cell pellet by adherence to plastic. The cell pellet was resuspended in $10 \mathrm{~mL}$ of 1:1 DMEM/Ham's F-12 containing $2 \%$ bovine serum. One aliquot of re-suspended AMs was preserved for GSH and GSSG analysis. Another aliquot was treated with protease inhibitors and immediately frozen at $-80^{\circ} \mathrm{C}$ for HDAC, HAT, and IL- 8 analysis. The other AMs were added to glass chamber slides containing $100 \mu \mathrm{L}$ medium with or without $500 \mu \mathrm{M} \mathrm{GSH}$. The AMs were incubated at $37^{\circ} \mathrm{C}$ with $10 \%$ carbon dioxide for $15 \mathrm{~h}$, after which fluorescein isothiocyanate-conjugated inactived Staphylococcus aureus (Invitrogen, Carlsbad, CA) was added in a 10:1 ratio and incubated for $2 \mathrm{~h}$. Cells were fixed with $3.7 \%$ para-formaldehyde and stored at $4^{\circ} \mathrm{C}$.

Urea nitrogen was measured in the plasma and BAL supernatant by a quantitative colorimetric assay (Pointe Scientific, Canton, MI) with a sensitivity of 0.05 to $150 \mathrm{mg} / \mathrm{dL}$. The dilution of the BAL was calculated from [urea $]_{\text {plasma }} /[\text { urea }]_{\text {BAL }}(18)$. The protein content of the BAL supernatant and AM cell lysate were determined using a Coomassie protein assay (Pierce Biotechnology, Rockford, IL) with a detection limit of $1 \mu \mathrm{g} / \mathrm{mL}$.

GSH and GSSG were measured in the BAL supernatant and AM lysate as previously described (2) by HPLC after derivatization of the samples with dansyl chloride (17). Derivatives were separated on a 10- $\mu$ m Ultrasil aminocolumn with fluorescence detection at $365 \mathrm{~nm}$ (Waters Alliance 2690; Waters Corporation, Milford, MA). GSH and GSSG were quantified relative to $\gamma$-glutamyl-glutamate by integration (19). Intracellular AM GSSG was also assessed in fixed AMs at baseline and after $500 \mu \mathrm{M}$ GSH supplementation by immunostaining for GSH protein complexes [1:100 anti-GSH (D8); Santa Cruz Biotechnology, Santa Cruz, CA]. Relative fluorescent units were determined by quantitative digital analysis from 10 fields using Image-Pro Plus for Windows (Version 3.1; Media Cybernetics, Inc., Silver Spring, MD).

To further characterize the extent of lipid and DNA oxidation in the BAL supernatant, malondialdehyde (MDA) concentrations were quantified using a commercially-available spectrophotometric assay (Bioxytech MDA-586; Oxis International, Foster City, CA) with a sensitivity of $1 \mu \mathrm{M}$. Free and DNA-incorportated 8-hydroxy-2-deoxyguanosine (8-OH-dg) concentrations were measured by competitive assay using a mouse IgG-coated plate and 8-OH-dg enzyme conjugate tracer with a detection limit of $30 \mathrm{pg} / \mathrm{mL}$ (Cayman Chemical, Ann Arbor, MI).
HDAC and HAT activities were measured in the AM cell lysate using commercially available assay kits (Abcam, Cambridge, MA; Cayman Chemical) at an excitation and emission of 360 and $450 \mathrm{~nm}$, respectively. IL- 8 was measured in the BAL supernatant and AM cell lysate by a bead-based assay with a sensitivity of $0.11 \mathrm{pg} / \mathrm{mL}$ (Millipore, Billerica, MA). Data were analyzed using the BioRad Bio-Plex System (Bio-Rad Laboratories, Hercules, CA) with gates of 4,335 and 10,000 .

AM phagocytosis was assessed as described previously (12) at baseline and after $500 \mu \mathrm{M}$ GSH supplementation using an Olympus confocal microscope (model BX51; Olympus America Inc.) containing an argon/krypton laser. AMs were analyzed from 10 separate fields by quantitative digital fluorescence imaging software (Olympus FluoView 300, Version 4.3). To ensure internalization of $S$. aureus, laser confocal microscopy was performed at $50 \%$ of the AM depth using identical background and gain settings. AMs with bacterial inclusions were considered positive for phagocytosis. A phagocytic index was calculated by multiplying the percentage of AMs undergoing phagocytosis by the average relative fluorescent units per phagocytic cell.

Apoptosis at baseline and after $500 \mu \mathrm{M}$ GSH supplementation was determined by immunostaining for cleavage of poly (ADP-ribose) polymerase (PARP) in fixed AMs using a 1:100 dilution of anti-PARP-1 (Santa Cruz Biotechnology). Cellular fluorescence was determined by quantitative digital analysis using Image-Pro Plus for Windows (Version 3.1; Media Cybernetics, Inc.). Data were expressed as the percentage of AMs fluorescently positive from 10 experimental fields per set.

Data analysis. Data analysis was performed with SPSS® software (Version 17; SPSS Inc., Chicago, IL). GSH, GSSG, MDA, 8-OH-dg, and IL-8 were logarithmically transformed given their nonnormal distribution. Data were expressed in the raw BAL fluid and per milliliter of ELF according to the urea dilution (18). AM lysate data were expressed per $\mu \mathrm{g}$ of protein. The $t$ tests and paired $t$ tests were performed for all between-group and within-group comparisons, respectively. Pearson correlation coefficients were also used to determine associations between variables. Two-tailed tests were used with significance defined as $\alpha<0.05$.

\section{RESULTS}

Twenty-one children with moderate asthma and 43 children with severe asthma were enrolled. Although five children with moderate asthma underwent bronchoscopy for recurrent pneumonia, these children were stable at the time of enrollment and had no evidence of active airway infection, including negative viral respiratory panels and no evidence of bacterial airway colonization. Bronchoscopy with BAL was well tolerated, and no child required overnight hospitalization or prolonged observation. Features of the groups are shown in Table 1. Children with severe asthma had increased markers of allergic sensitization and were more likely to be of African American descent. Children with severe asthma also had increased airflow obstruction and greater healthcare utilization despite more aggressive corticosteroid treatment.

GSH homeostasis, oxidative stress, and inflammation in the $\boldsymbol{E} \boldsymbol{L F}$. There were no significant differences in BAL or ELF GSH concentrations between groups. Although there were no significant differences in the total BAL or ELF GSH + GSSG pool between groups (BAL: median 1123 versus $704 \mathrm{nmol} / \mathrm{L}$, $p=0.539$; ELF: median 64 versus $57 \mu \mathrm{M}, p=0.207$ ), children with severe asthma had significantly higher BAL and ELF concentrations of GSSG (Table 2). This was accompanied by increased lipid peroxidation and increased DNA oxidation as measured by MDA and $8-\mathrm{OH}-\mathrm{dg}$, respectively (Table 2). IL-8 was also increased in children with severe asthma (Table 2) and was strongly associated with the percentage of airway neutrophils $(r=0.452, p=0.001)$.

GSH homeostasis and inflammation in AMs. The imbalance in airway GSH homeostasis was also reflected at the cellular level. Although intracellular AM concentrations of 
Table 1. Features of children with moderate $(\mathrm{n}=21)$ and severe $(\mathrm{n}=43)$ asthma

\begin{tabular}{|c|c|c|c|}
\hline Feature & $\begin{array}{l}\text { Moderate } \\
\text { asthma }\end{array}$ & $\begin{array}{l}\text { Severe } \\
\text { asthma }\end{array}$ & $p$ \\
\hline Age (y) & $11 \pm 4$ & $10 \pm 4$ & 0.339 \\
\hline Male & $12(57)$ & $22(51)$ & 0.428 \\
\hline African-American & $3(14)$ & $22(51)$ & 0.004 \\
\hline $\begin{array}{l}\text { ICS dose ( } \mu \mathrm{g} \text { fluticasone } \\
\text { equivalents/d) }\end{array}$ & $351 \pm 287$ & $810 \pm 299$ & $<0.001$ \\
\hline \multicolumn{4}{|l|}{ Asthma medications } \\
\hline Fluticasone & $2(10)$ & $2(5)$ & 0.398 \\
\hline Fluticasone/salmeterol & $8(38)$ & $31(72)$ & 0.010 \\
\hline Montelukast & $12(57)$ & $38(88)$ & 0.007 \\
\hline Prednisone & 0 & $17(40)$ & $<0.001$ \\
\hline \multicolumn{4}{|l|}{ Asthma medical history } \\
\hline History of asthma in parent & $8(38)$ & $29(67)$ & 0.025 \\
\hline Aeroallergen sensitization* & $16(76)$ & $35(81)$ & 0.334 \\
\hline Atopic dermatitis & $4(19)$ & $25(58)$ & 0.003 \\
\hline $\begin{array}{l}\text { Hospitalization (previous } \\
\text { year) }\end{array}$ & $2(10)$ & $31(72)$ & $<0.001$ \\
\hline Intubation (ever) & 0 & $7(16)$ & 0.052 \\
\hline Serum $\operatorname{IgE}(\mathrm{kU} / \mathrm{L}) \dagger$ & $22(3-600)$ & $326(2-3946)$ & 0.007 \\
\hline \multicolumn{4}{|l|}{ Pulmonary function } \\
\hline FVC (\% predicted) & $95 \pm 16$ & $91 \pm 21$ & 0.513 \\
\hline $\mathrm{FEV}_{1}(\%$ predicted $)$ & $94 \pm 16$ & $80 \pm 23$ & 0.009 \\
\hline $\mathrm{FEV}_{1}: \mathrm{FVC}$ & $0.87 \pm 0.08$ & $0.76 \pm 0.12$ & $<0.001$ \\
\hline $\mathrm{FEF}_{25-75}(\%$ predicted $)$ & $100 \pm 35$ & $61 \pm 29$ & $<0.001$ \\
\hline \multicolumn{4}{|l|}{ BAL cellular differential (\%) } \\
\hline Macrophages/monocytes & $91 \pm 5$ & $88 \pm 6$ & 0.102 \\
\hline Neutrophils & $4 \pm 3$ & $6 \pm 5$ & 0.452 \\
\hline Eosinophils & $1 \pm 1$ & $2 \pm 3$ & 0.352 \\
\hline Lymphocytes & $4 \pm 3$ & $4 \pm 3$ & 0.948 \\
\hline
\end{tabular}

Data are expressed as the mean \pm SD or frequency (\%) unless otherwise stated. FEF, forced expiratory flow (between $25 \%$ and $75 \%$ of forced vital capacity).

* As determined by skin prick or specific IgE testing.

$\dagger$ Data are expressed as the median (range).

GSH were not different between groups $(175 \pm 120 \mathrm{nmol} / \mathrm{L}$ versus $190 \pm 255 \mathrm{nmol} / \mathrm{L}$ for severe versus moderate asthma, $p=0.283$ ), children with severe asthma had higher intracellular GSSG concentrations with an accompanying increase in the percentage of GSSG in the total intracellular GSH + GSSG pool (Fig. 1). Within the severe asthma group, stratification by high intracellular GSSG $(>250 \mathrm{nM} / \mu \mathrm{g}$ protein, $n=7)$ versus low intracellular GSSG $(<250 \mathrm{nM} / \mu \mathrm{g}$ protein, $n=16)$ revealed no significant differences in age, gender, race, ICS dose, asthma medical history, healthcare utilization, and lung function or airway cellularity between groups, suggesting that clinical features alone were not responsible for the results. However, intracellular GSSG concentrations did correlate with GSSG concentrations in the BAL supernatant ( $r=$ $0.375, p=0.038)$ and $\operatorname{ELF}(r=0.322, p=0.05)$. No associations between intracellular GSSG and the oxidative biomarkers MDA or 8-OH-dg were observed.

AM HAT activities were not different between groups $(10.1 \pm 9.5$ versus $8.7 \pm 5.5$ for severe versus moderate asthma, $p=0.657$ ). However, AM HDAC activities were significantly blunted in children with severe asthma, suggesting increased inflammatory gene transcription (Fig. 2). IL-8 was also significantly up-regulated in AMs from children with severe asthma (Fig. 2) and further correlated with AM HDAC activity $(r=-0.618, p=0.006)$ and IL-8 concentrations in the BAL supernatant $(r=0.830, p<0.001)$ and $\operatorname{ELF}(r=$ $0.748, p=0.002$ ).

AM function and effect of GSH supplementation. In children with severe asthma, the shift in the ratio of AM intracellular GSH to GSSG was accompanied by impairment of AM function. Compared with children with moderate asthma, children with severe asthma had significantly blunted phagocytosis of $S$. aureus and increased apoptosis as measured by cleavage of PARP-1 (Fig. 2). The degree of AM phagocytic impairment was strongly associated with absolute GSSG concentrations and the percentage of GSSG in the total GSH + GSSG pool in both the ELF and within the AM intracellular space (Fig. 3). AM apoptosis also correlated with ELF GSSG concentrations $(r=0.310, p=0.05)$ and the percentage of ELF GSSG $(r=0.451, p=0.035)$. Ex vivo supplementation with $500 \mu \mathrm{M}$ GSH, a physiologically relevant concentration of GSH that is commonly observed in the ELF of healthy controls (4), significantly increased AM phagocytosis and reduced AM apoptosis in children with severe asthma (Fig. 4). In children with severe asthma $(n=5)$, GSH supplementation further reduced intracellular GSSG as reflected by a significant decrease in GSH protein complexes $(8627 \pm 505 \mathrm{RFU}$ versus $5574 \pm 766 \mathrm{RFU}$ for baseline versus $\mathrm{GSH}, p=0.015$ ).

\section{DISCUSSION}

This study is the first to quantify the relationship between extracellular and intracellular GSH homeostasis and AM function in children with severe asthma. Although extracellular GSH concentrations were not different in the airways of children with moderate and severe asthma, children with severe asthma had significantly higher GSSG concentrations accompanied by increased lipid peroxidation and increased by-products of DNA oxidation in the extracellular space. This shift in the airway GSH to GSSG ratio was also mirrored within the AMs and was further associated with AM functional disturbances, including decreased suppression of histone acetylation, impairment of phagocytosis, and increased apoptosis. Ex vivo supplementation with GSH improved phagocytosis and decreased apoptosis in children with severe asthma while also decreasing intracellular GSSG, suggesting that airway GSH and GSSG are the primary regulators of AM function in these children. Thus, airway GSH homeostasis may represent a novel target for therapeutic intervention in children with severe asthma.

GSH is a tripeptide thiol that is essential for defense against oxidative damage and function of airway cells. Depletion of GSH through either a shift in the ratio of GSH to GSSG or a reduction in the total GSH + GSSG pool results in activation of nuclear factor kappa B (NF-kappaB) and pro-inflammatory cytokine release from HDAC suppression and increased gene transcription $(8,20)$. GSH depletion has also been linked to increased AM apoptosis from caspase activation and PARP degradation (21) and to impaired phagocytosis of infectious particles (22), perhaps through inhibition of the transcription factor, NF-E2-related factor 2 (Nrf2) (11). Although molecular mechanisms of altered GSH homeostasis have not been studied in patients with asthma, our findings of increased 
Table 2. Concentrations of airway fluid biomarkers in children with moderate $(\mathrm{n}=20)$ and severe $(\mathrm{n}=35)$ asthma

\begin{tabular}{|c|c|c|c|c|c|c|}
\hline \multirow[b]{2}{*}{ Biomarker } & \multicolumn{3}{|c|}{ Raw concentration in the BAL fluid supernatant } & \multicolumn{3}{|c|}{ Concentration per $\mathrm{mL}$ of ELF } \\
\hline & $\begin{array}{l}\text { Moderate } \\
\text { asthma }\end{array}$ & $\begin{array}{l}\text { Severe } \\
\text { asthma }\end{array}$ & $\begin{array}{c}p \text { value } \\
\text { for BAL* }\end{array}$ & $\begin{array}{l}\text { Moderate } \\
\text { asthma }\end{array}$ & $\begin{array}{l}\text { Severe } \\
\text { asthma }\end{array}$ & $\begin{array}{l}p \text { value } \\
\text { for ELF* }\end{array}$ \\
\hline GSH (nmol/L BAL, $\mu \mathrm{mol} / \mathrm{L}$ ELF) & $334(36-1352)$ & $262(7-1510)$ & 0.628 & $37(3-480)$ & $24(2-178)$ & 0.292 \\
\hline GSSG (nmol/L BAL, $\mu \mathrm{mol} / \mathrm{L}$ ELF) & $134(26-929)$ & $447(42-3113)$ & 0.006 & $13(3-71)$ & $32(2-201)$ & 0.015 \\
\hline MDA (nmol/L BAL, $\mu \mathrm{mol} / \mathrm{L}$ ELF) & $565(165-980)$ & $860(165-3563)$ & 0.039 & $37(8-104)$ & $54(11-162)$ & 0.037 \\
\hline 8-OH-dg (pg/mL BAL, ng/mL ELF) & $168(29-667)$ & $537(30-5056)$ & 0.011 & $13(2-231)$ & $24(3-650)$ & 0.031 \\
\hline IL-8 (pg/mL BAL, ng/mL ELF) & $15(4-104)$ & $32(6-1984)$ & 0.042 & $2(0-4)$ & $3(1-137)$ & 0.050 \\
\hline
\end{tabular}

Data are expressed as the median (range).

* After logarithmic transformation.
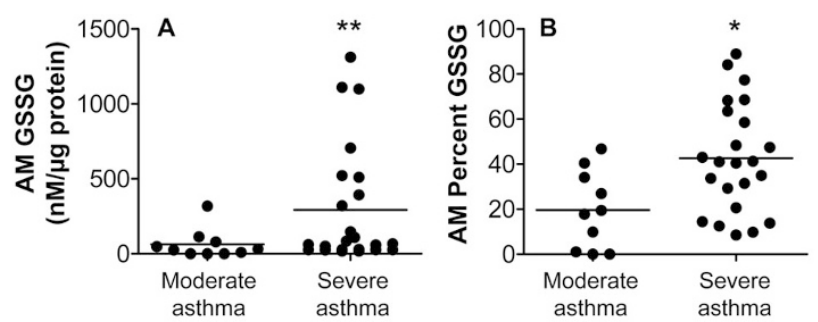

Figure 1. (A) Intracellular GSSG concentrations and $(B)$ the percentage of intracellular GSSG in the total GSH + GSSG pool in AMs from children with severe $(n=23)$ vs moderate $(n=10)$ asthma. Lines represent the mean. $* p<$ $0.05 ; * * p<0.01$.
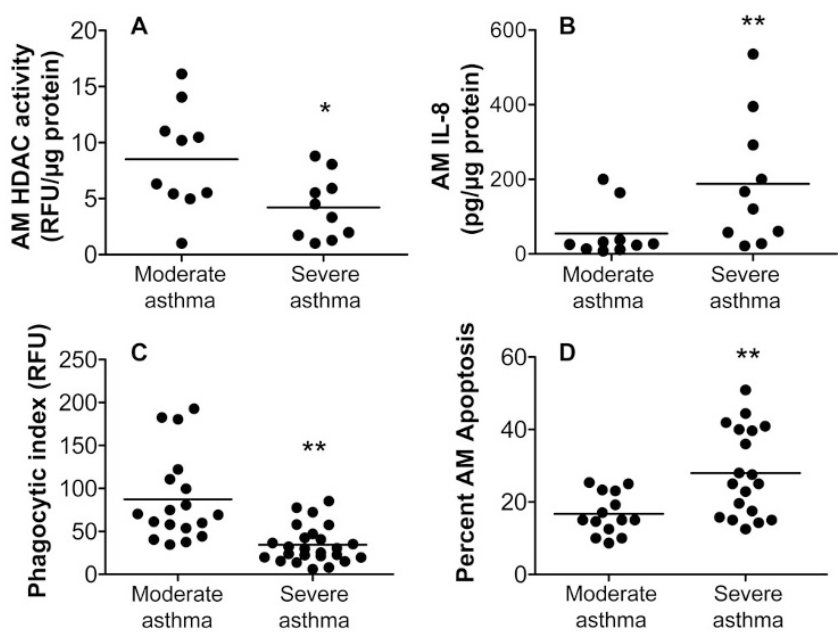

Figure 2. AM $(A)$ HDAC activity, $(B)$ IL-8, $(C)$ phagocytosis of $S$. aureus, and $(D)$ apoptosis in children with severe vs moderate asthma. $(A)$ and $(B)$, $n=10$ per group; $(C) n=26$ vs 20 for severe $v$ moderate asthma; $(D), n=$ $19 v s 14$ for severe $v s$ moderate asthma. Lines represent the mean. $* p<0.05$, $* * p \leq 0.01$.

intracellular GSSG and corresponding AM dysfunction in the form of decreased HDAC activity, increased apoptosis, and impaired phagocytosis suggest that GSH and perhaps NFkappaB and Nrf2 may regulate many features of the disorder. Indeed, in murine models of allergic airway inflammation, GSH attenuates airway hyperresponsiveness and the airway Th2 imbalance through suppression of chemokine-induced eosinophil chemotaxis and inhibition of NF-kappaB $(9,23,24)$. Similarly, although Nrf2-deficient mice have increased susceptibility to acute lung injury, GSH supplementation prevents lung injury in Nrf2-deficient mice by inhibiting the recruitment of inflammatory cells to the site of airway injury (25).
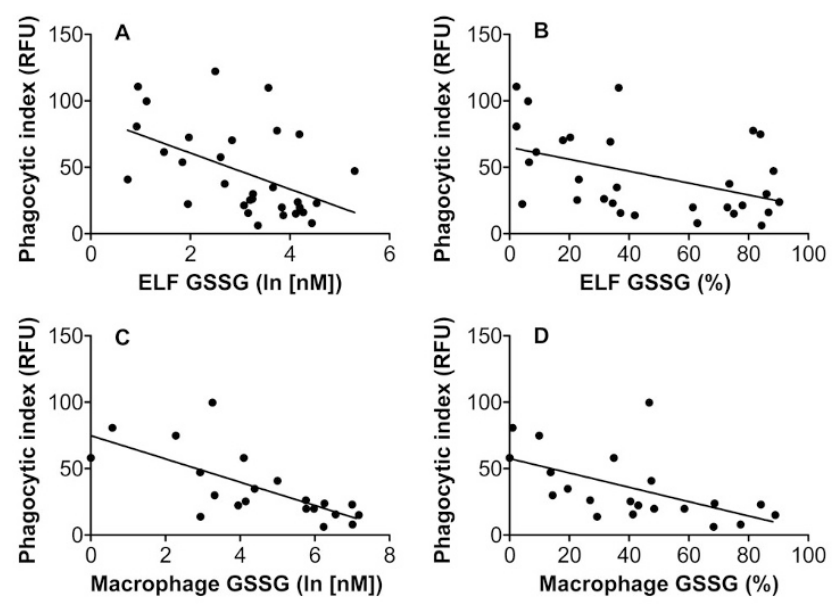

Figure 3. Association between the AM phagocytic index and (A) ELF GSSG concentrations $(r=-0.49, p<0.01),(B)$ the percentage of GSSG in the ELF $(r=-0.45, p<0.05),(C)$ AM intracellular GSSG concentrations $(r=$ $-0.71, p<0.001)$, and $(D)$ the percentage of intracellular AM GSSG $(r=$ $-0.56, p<0.01)$. The phagocytic index is expressed in relative fluorescent units.
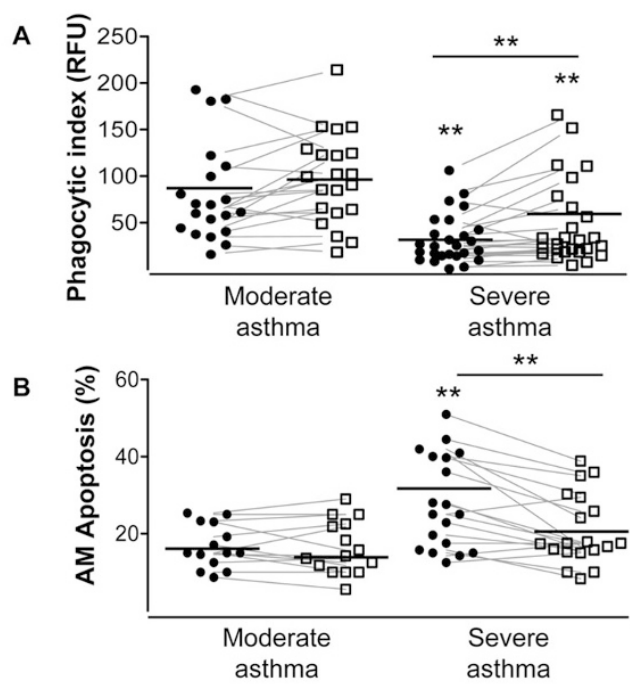

Figure 4. AM $(A)$ phagocytosis and $(B)$ apoptosis in children with severe $v s$ moderate asthma before $(\bullet)$ and after $500 \mu \mathrm{M} \mathrm{GSH}(\square)$. (A) $n=26$ vs 20 for severe $v s$ moderate asthma; $(B) n=19 v s 15$ for severe $v s$ moderate asthma. Lines represent the mean. ${ }^{*} p<0.05,{ }^{*} p<0.01$.

These data highlight the important role of GSH in the airways and argue for additional study of the mechanisms of GSHrelated airway dysfunction in severe asthma. 
Although we did not observe differences in ELF GSH concentrations between children with moderate and severe asthma in this study ( $\sim 30 \mu \mathrm{M}$ in each group), these levels are significantly lower than those previously reported in healthy adults $(500 \mu \mathrm{M})$ and in our previous studies of nonasthmatic children with chronic cough $(250 \mu \mathrm{M})(2,4)$. Although it is possible that these decreased GSH concentrations are due to a defect in GSH-related enzymatic activities or polymorphisms in GSH transferases, we recently observed no differences in the activity of GSH peroxidase, GSH-S-transferase, or GSH reductase between children with moderate and severe asthma and healthy controls (2). Thus, the GSH concentrations that we observed here are likely related to chronic airway oxidation and diffuse airway injury. Indeed, the ELF GSH concentrations that we observed in children with moderate and severe asthma are consistent with those of adults with acute respiratory distress syndrome, a disorder associated with diffuse alveolar injury $(26,27)$. Although asthma is traditionally thought to affect the bronchial versus the alveolar airspaces, there is increasing evidence of distal airway inflammation in both humans $(28,29)$ and in experimental models of asthma (30). Although our BAL samples were pooled and do include a mixture of both bronchial and lower airway constituents, our data may reflect early distal airway damage in moderate to severe asthmatic children that is reflected by low GSH concentrations and altered GSH homeostasis.

This study does have limitations. Because, it is not ethical to perform bronchoscopy for research purposes in healthy children, it is unclear how our findings relate to steroid-naïve children with mild asthma or children with mild asthma that is well controlled with low doses of ICS. Furthermore, it is possible that intracellular and extracellular GSH and GSSG concentrations and AM function could be influenced by asthma treatment. However, we do not believe that corticosteroid use sufficiently explains the discrepancies observed in this study. Given the magnitude of symptoms in children with moderate asthma, all children in this study were treated with ICS. Furthermore, there were no significant correlations between corticosteroid usage and the oxidative and AM functional parameters measured in this study.

In summary, severe asthma in children is a complicated disorder with persistent airway inflammation and ongoing symptoms that are refractory to treatment with high doses of inhaled and oral corticosteroids. This airway inflammation is further accompanied by AM cellular dysfunction, including increased apoptosis and impaired AM phagocytosis of infectious particles. Our findings of altered intracellular and extracellular airway GSH homeostasis provide a possible mechanism for these alterations. Given the vital role of GSH in airway antioxidant defense and cellular function, airway GSH warrants further study in children with severe asthma. Interventions to improve airway GSH homeostasis may ultimately be needed to decrease the morbidity of severe asthma in children.

Acknowledgments. The Severe Asthma Research Program (SARP) is a multicenter asthma research group funded by the NHLBI and consisting of the following contributors (Principal Investigators are marked with an asterisk): Brigham \& Women's
Hospital—Elliot Israel*, Bruce D. Levy, Michael E. Wechsler, Shamsah Kazani, and Gautham Marigowda; Cleveland ClinicSerpil C. Erzurum*, Raed A. Dweik, Suzy A.A. Comhair, Emmea Cleggett-Mattox, Deepa George, Marcelle Baaklini, and Daniel Laskowski; Emory University—Anne M. Fitzpatrick, Denise Whitlock, and Shanae Wakefield; Imperial College School of Medicine-Kian Fan Chung*, Mark Hew, Patricia Macedo, Sally Meah, and Florence Chow; University of IowaEric Hoffman*, Janice Cook-Granroth; University of Pittsburgh-Sally E. Wenzel*, Fernando Holguin, Silvana Balzar, and Jen Chamberlin; University of Texas-Medical BranchWilliam J. Calhoun* and Bill T. Ameredes; University of Virginia—Benjamin Gaston*, W. Gerald Teague*, and Denise Thompson-Batt; University of Wisconsin-William W. Busse*, Nizar Jarjour, Ronald Sorkness, Sean Fain, and Gina Crisafi; Wake Forest University—Eugene R. Bleecker*, Deborah Meyers, Wendy Moore, Stephen Peters, Rodolfo M. Pascual, Annette Hastie, Gregory Hawkins, Jeffrey Krings, and Regina Smith; Washington University in St. Louis-Mario Castro*, Leonard Bacharier, and Jaime Tarsi; Data Coordinating Center-Douglas Curran-Everett*, Ruthie Knowles, Maura Robinson, and Lori Silveira; NHLBI—Patricia Noel, Robert Smith.

\section{REFERENCES}

1. Fitzpatrick AM, Gaston BM, Erzurum SC, Teague WG 2006 Features of severe asthma in school-age children: atopy and increased exhaled nitric oxide. J Allergy Clin Immunol 118:1218-1225

2. Fitzpatrick AM, Teague WG, Holguin F, Yeh M, Brown LA. Severe Asthma Research Program 2009 Airway glutathione homeostasis is altered in children with severe asthma: evidence for oxidant stress. J Allergy Clin Immunol 123:146-152.e8

3. Comhair SA, Ricci KS, Arroliga M, Lara AR, Dweik RA, Song W, Hazen SL, Bleecker ER, Busse WW, Chung KF, Gaston B, Hastie A, Hew M, Jarjour N, Moore W, Peters S, Teague WG, Wenzel SE, Erzurum SC 2005 Correlation of systemic superoxide dismutase deficiency to airflow obstruction in asthma. Am J Respir Crit Care Med 172:306-313

4. Cantin AM, North SL, Hubbard RC, Crystal RG 1987 Normal alveolar epithelial lining fluid contains high levels of glutathione. J Appl Physiol 63:152-157

5. Rahman I, Mulier B, Gilmour PS, Watchorn T, Donaldson K, Jeffery PK, MacNee W 2001 Oxidant-mediated lung epithelial cell tolerance: the role of intracellular glutathione and nuclear factor-kappab. Biochem Pharmacol 62:787-794

6. Rahman I, Yang SR, Biswas SK 2006 Current concepts of redox signaling in the lungs. Antioxid Redox Signal 8:681-689

7. Kloek J, Mortaz E, van Ark I, Lilly CM, Nijkamp FP, Folkerts G 2010 Glutathione prevents the early asthmatic reaction and airway hyperresponsiveness in guinea pigs. J Physiol Pharmacol 61:67-72

8. Rahman I, Gilmour PS, Jimenez LA, MacNee W 2002 Oxidative stress and tnf-alpha induce histone acetylation and nf-kappab/ap-1 activation in alveolar epithelial cells: potential mechanism in gene transcription in lung inflammation. Mol Cell Biochem 234-235:239-248

9. Koike Y, Hisada T, Utsugi M, Ishizuka T, Shimizu Y, Ono A, Murata Y, Hamuro J, Mori M, Dobashi K 2007 Glutathione redox regulates airway hyperresponsiveness and airway inflammation in mice. Am J Respir Cell Mol Biol 37:322-329

10. Corradi M, Folesani G, Andreoli R, Manini P, Bodini A, Piacentini G, Carraro S, Zanconato S, Baraldi E 2003 Aldehydes and glutathione in exhaled breath condensate of children with asthma exacerbation. Am J Respir Crit Care Med 167:395-399

11. Reddy NM, Suryanarayana V, Kalvakolanu DV, Yamamoto M, Kensler TW, Hassoun PM, Kleeberger SR, Reddy SP 2009 Innate immunity against bacterial infection following hyperoxia exposure is impaired in nrf2-deficient mice. J Immunol 183:4601-4608

12. Fitzpatrick AM, Holguin F, Teague WG, Brown LA 2008 Alveolar macrophage phagocytosis is impaired in children with poorly controlled asthma. J Allergy Clin Immunol 121:1372-1378, 1378.e1-e3

13. National Asthma Education and Prevention Program 2007 Expert panel report 3 (EPR-3): guidelines for the diagnosis and management of asthma-summary report 2007. J Allergy Clin Immunol 120:S94-S138

14. Wenzel SE, Fahy JV, Irvin C, Peters SP, Spector S, Szefler SJ. for the American Thoracic Society Workshop 2000 Proceedings of the ATS workshop on refractory asthma: current understanding, recommendations, and unanswered questions. American Thoracic Society. Am J Respir Crit Care Med 162:2341-2351

15. Miller MR, Hankinson J, Brusasco V, Burgos F, Casaburi R, Coates A, Crapo R, Enright P, van der Grinten CP, Gustafsson P, Jensen R, Johnson DC, MacIntyre N, McKay R, Navajas D, Pedersen OF, Pellegrino R, Viegi G, Wanger J 2005 Standardisation of spirometry. Eur Respir J 26:319-338 
16. Wang X, Dockery DW, Wypij D, Fay ME, Ferris BG Jr 1993 Pulmonary function between 6 and 18 years of age. Pediatr Pulmonol 15:75-88

17. Jones DP, Carlson JL, Samiec PS, Sternberg P Jr, Mody VC Jr, Reed RL, Brown LA 1998 Glutathione measurement in human plasma. Evaluation of sample collection, storage and derivatization conditions for analysis of dansyl derivatives by HPLC. Clin Chim Acta 275:175-184

18. Rennard SI, Basset G, Lecossier D, O'Donnell KM, Pinkston P, Martin PG, Crystal RG 1986 Estimation of volume of epithelial lining fluid recovered by lavage using urea as marker of dilution. J Appl Physiol 60:532-538

19. Reed DJ, Babson JR, Beatty PW, Brodie AE, Ellis WW, Potter DW 1980 Highperformance liquid chromatography analysis of nanomole levels of glutathione, glutathione disulfide, and related thiols and disulfides. Anal Biochem 106:55-62

20. Yang SR, Chida AS, Bauter MR, Shafiq N, Seweryniak K, Maggirwar SB, Kilty I, Rahman I 2006 Cigarette smoke induces proinflammatory cytokine release by activation of nf-kappab and posttranslational modifications of histone deacetylase in macrophages. Am J Physiol Lung Cell Mol Physiol 291:L46-L57

21. Hu S, Zhao H, Al-Humadi NH, Yin XJ, Ma JK 2006 Silica-induced apoptosis in alveolar macrophages: evidence of in vivo thiol depletion and the activation of mitochondrial pathway. J Toxicol Environ Health A 69:1261-1284

22. Brown LA, Ping XD, Harris FL, Gauthier TW 2007 Glutathione availability modulates alveolar macrophage function in the chronic ethanol-fed rat. Am J Physiol Lung Cell Mol Physiol 292:L824-L832

23. Lee KS, Kim SR, Park HS, Park SJ, Min KH, Lee KY, Choe YH, Hong SH, Han HJ, Lee YR, Kim JS, Atlas D, Lee YC 2007 A novel thiol compound, n-acetylcysteine amide, attenuates allergic airway disease by regulating activation of nf-kappab and hypoxia-inducible factor-1alpha. Exp Mol Med 39:756-768

24. Lowry MH, McAllister BP, Jean JC, Brown LA, Hughey RP, Cruikshank WW, Amar S, Lucey EC, Braun K, Johnson P, Wight TN, Joyce-Brady M 2008 Lung lining fluid glutathione attenuates il-13-induced asthma. Am J Respir Cell Mol Biol 38:509-516

25. Reddy NM, Kleeberger SR, Kensler TW, Yamamoto M, Hassoun PM, Reddy SP 2009 Disruption of nrf2 impairs the resolution of hyperoxia-induced acute lung injury and inflammation in mice. J Immunol 182:7264-7271

26. Bunnell E, Pacht ER 1993 Oxidized glutathione is increased in the alveolar fluid of patients with the adult respiratory distress syndrome. Am Rev Respir Dis 148:11741178

27. Schmidt R, Luboeinski T, Markart P, Ruppert C, Daum C, Grimminger F, Seeger W Gunther A 2004 Alveolar antioxidant status in patients with acute respiratory distress syndrome. Eur Respir J 24:994-999

28. Balzar S, Chu HW, Strand M, Wenzel S 2005 Relationship of small airway chymase-positive mast cells and lung function in severe asthma. Am J Respir Crit Care Med 171:431-439

29. Fitzpatrick AM, Brown LA, Holguin F, Teague WG; National Institutes of Health/ National Heart, Lung, and Blood Institute Severe Asthma Research Program 2009 Levels of nitric oxide oxidation products are increased in the epithelial lining fluid of children with persistent asthma. J Allergy Clin Immunol 124:990-996.e1-e9

30. Wegmann M, Fehrenbach H, Fehrenbach A, Held T, Schramm C, Garn H, Renz H 2005 Involvement of distal airways in a chronic model of experimental asthma. Clin Exp Allergy 35:1263-1271 